\title{
The Wireless Sensor Networks Base Layout and Density Optimization Oriented towards Traffic Information Collection
}

\author{
Musong Gu, ${ }^{1,2}$ Lei You, ${ }^{1}$ Jun Hu, ${ }^{3,4}$ Lintao Duan, ${ }^{1}$ and Zhen Zuo ${ }^{5}$ \\ ${ }^{1}$ College of Information Science and Technology, Chengdu University, Chengdu 610106, China \\ ${ }^{2}$ Key Laboratory of Pattern Recognition and Intelligent Information Processing, Institutions of Higher Education of Sichuan Province, \\ Chengdu 610106, China \\ ${ }^{3}$ Faculty of Computer Science, Chengdu Normal University, Chengdu 611130, China \\ ${ }^{4}$ School of Traffic and Transportation, Beijing Jiaotong University, Beijing 100044, China \\ ${ }^{5}$ Unocal East China Sea, Co. Ltd., Chengdu 610012, China \\ Correspondence should be addressed to Jun Hu; junhu_cdu@163.com
}

Received 26 November 2014; Accepted 29 March 2015

Academic Editor: Roman Lewandowski

Copyright (c) 2015 Musong Gu et al. This is an open access article distributed under the Creative Commons Attribution License, which permits unrestricted use, distribution, and reproduction in any medium, provided the original work is properly cited.

\begin{abstract}
Wireless sensor networks (WSN) are applied in Intelligent Transport System for data collection. For the low redundancy rate of the wireless sensor networks nodes of traffic information collection, the senor nodes should be deployed reasonably for the WSN nodes to work effectively, and, thus, the base network structure and the density optimization of the sensor network are one of the main problems of WSN application. This paper establishes the wireless sensor networks design optimization model oriented to the traffic information collection, solving the design optimization model with the chemical reaction optimization (CRO) algorithm. The experimental results show that CRO algorithm outperforms the traditional particle swarm optimization (PSO) in solving the wireless sensor network design optimization oriented to the traffic information collection, capable of optimizing the wireless sensor network deployment of traffic information collection to contribute to the great improvement of the comprehensive value of the network performance. The reasonable design of the wireless sensor network nodes has great significance for the information collection, post-maintenance-and-extension, and cost saving of a monitoring system.
\end{abstract}

\section{Introduction}

The wireless sensor network is a brand new information collection and processing technology. Compared with the various technologies of the present traffic monitoring system, the wireless sensor network boasts the advantage of being unrestricted by the environment, self-organizing, and distribution. And, thus, WSN is now tentatively applied in the intelligent traffic system [1] for data collection. With the low redundancy rate of the sensor nodes in the traffic monitoring system [2], reasonable deployment of the sensor nodes should be made to enable WSN to function more effectively, and, thus, the base network structure composition and density optimization $[3,4]$ are one of the main problems of WSN application.

Most of the researches on WSN both domestic and overseas are conducted in the monitoring area where nodes are spilled randomly and multiple optimization methods are employed for nodes distribution such as virtual force oriented particle swarm strategy [5], genetic algorithms [6], and simulated annealing algorithm [7]. In [8], an approximate algorithm is applied to solve the sensor distribution integral linear planning problem for the purpose of the lowest cost based on the satisfaction of coverage. Literature [9] applies Particle Swarm Algorithm to study the coverage of the wireless sensor networks.

Although the existing research results have provided the optimization method of the sensor network node deployment, they take no account of the impact on the perception coverage by the intercommunication among sensors. In the paper, according to the requirement of the traffic information collection for the sensor, the comprehensive evaluation function of coverage and connectivity of the wireless sensor network nodes is used; for the reasonable deployment of the 
sensor network nodes, the multiple constrained optimization mathematical model is established, which is converted into the single constrained optimization problem by means of the geometric weighting method, solving the problem by means of the chemical reaction optimization (CRO) [10-13].

\section{Problem Description}

WSN consists of management grids, sink nodes, and wireless sensor nodes. The management nodes are set in the regional control room or cabinet; the sink nodes are set around roadside traffic signal controller and cabinet; the wireless sensor nodes are buried on road surface or roadside. The traffic information collected by the wireless sensor nodes is transmitted hop by hop to the sink nodes along other sensor nodes. The sink nodes integrate the data of the wireless sensor nodes to obtain the data of the region or a small area, communicating with the management nodes through the Ethernet. The management nodes receive the data transmitted by the sink nodes to make an integration and comprehensive application of a large-scale regional data.

For the characteristics of the traffic information acquisition system and the wireless sensor networks, the following provisions are made for studying the problem:

(1) The starting point and the ending point of the simulation road section are known; there are $N$ wireless sensor nodes, $M$ sink nodes, and one management node.

(2) The number of sensor nodes set on the road is relevant to the length of the road and the distance of the sensor communication; depending on the traffic information collection need and the experience of experts, the position of sink nodes is determined around the signal or in the cabinet.

\section{Wireless Sensor Networks Coverage Problem}

In building the wireless sensor networks, the network coverage is one of the standard problems of the wireless sensor networks, which is concerning how to deploy the sensor network nodes to reach the biggest network coverage with as few network nodes as possible while guaranteeing the quality of service to some degree. Priority should be given to the node coverage rate and the regional coverage rate in the optimization of network coverage of the wireless sensor.

The node coverage rate: we assume that the monitored two-dimensional area $A$ is distributed into $m \times n$ meter by figure, where the number of sensor nodes with the same parameter launched is $N$, the coordinate of every node is known, the perception radius is $r$, and the communication radius is $R$. To ensure the connectivity of the network, the communication radius $R$ is usually bigger than the sensor radius $r$. Meanwhile, the communication effect may be influenced by the wireless disturbance if the communication radius $R$ is too big. According to experimental results, it achieves the best communication effect when $R$ equals $2 r$. $s_{i}=\left(x_{i}, y_{i}, r\right)$ is applied to indicate the circle with the node coordinate $\left\{x_{i}, y_{i}\right\}$ as the center and the monitored radius as $r$. We assume the pixel coordinate is $(x, y)$; then the distance between the target pixel and the sensor node is $d\left(s_{i}, p\right)=$ $\sqrt{\left(x_{i}-x\right)^{2}+\left(y_{i}-y\right)^{2}}$. The case of the pixel being covered by the sensor nodes is defined as $r_{i}$; then the probability of the case occurring is the same as that of the pixel $(x, y)$ being covered by the sensor node $S_{i}$; considering the monitored environment and the noise interruption [14], the measuring model of the sensor node shows the probability distribution with certain characteristics in actual application, which is

$$
\begin{aligned}
& p_{\text {cov }}\left(x, y, s_{i}\right) \\
& = \begin{cases}1 & d\left(s_{i}, p\right) \leq r-r_{e} \\
e\left(\frac{-a_{1} \lambda_{1} \beta_{1}}{\lambda_{2} \beta_{2}}\right) & r-r_{e} \leq d\left(s_{i}, p\right) \leq r+r_{e} \\
0 & \text { otherwise. }\end{cases}
\end{aligned}
$$

In the formula, $r_{e}\left(0<r_{e}<r\right)$ is the reliability parameter of the sensor node measurement, $\alpha_{1}, \alpha_{2}, \beta_{1}$, and $\beta_{2}$ are the measuring parameters relevant to the characteristics of sensor nodes, and $\lambda_{1}$ and $\lambda_{2}$ are the input parameters:

$$
\begin{aligned}
& \lambda_{1}=r-r_{e}+d\left(s_{i}, p\right) \\
& \lambda_{2}=r+r_{e}+d\left(s_{i}, p\right) .
\end{aligned}
$$

Communication model: to analyze the network performance, we adopt probability model as the communication model of the wireless sensor node $S_{i}$, reflecting the communication quality between nodes in actual network environment:

$$
T\left(S_{i}, S_{j}\right)= \begin{cases}1 & 0 \leq d\left(S_{i}, S_{j}\right) \\ e^{-\theta\left(d\left(S_{i}, S_{j}\right)-R_{1}\right)} & R_{1}<d\left(S_{i}, S_{j}\right) \leq R_{2} \\ 0 & d\left(S_{i}, S_{j}\right)>R_{2} .\end{cases}
$$

In the formula, $T\left(S_{i}, S_{j}\right)$ is the communication strength between the sensor nodes $S_{i}$ and $S_{j} . R_{1}$ is effective communication range, $R_{2}$ is the communication range value of signal attenuation, and we set $R_{1}=50 \mathrm{~m}$ and $R_{2}=100 \mathrm{~m}$. $\theta$ is the physical parameter of the sensor nodes, indicating the degree of attenuation of the communication strength as the distance increases; we set $\theta=1$.

\section{Optimization Model of Wireless Sensor Networks Deployment of Traffic Information Collection}

Evaluation function: the position vector of all sensor nodes in the wireless sensor network is indicated as the $N$-dimensional vector, in which $X=\left[x_{1}, x_{2}, \ldots, x_{N}\right]$ is the vector of the sensor node coordinate.

(a) Coverage Redundancy. Take the mean value of the probability of all the nodes being detected on the road, indicating the coverage performance of the sensor networks. In order to 
improve the target measurement probability, multiple sensor nodes are needed to measure the target. The joint probability is as follows:

$$
P_{\mathrm{cov}}(S)=1-\prod_{s_{i} \in S}\left(1-P_{\mathrm{cov}}\left(x, y, s_{i}\right)\right) .
$$

(b) Regional Coverage Rate. There is $m \times n$ meter in the monitored region $A$. The node set and the measuring probability $P_{\text {cov }}(S)$ are used to measure whether every pixel is covered. In the paper, the regional coverage rate of the node set $S$ is defined as the ratio of the coverage area of the node set $S$ to the total area of the monitored area $A$, which is

$$
R_{\text {area }}(S)=\frac{\sum P_{\text {cov }}(S)}{m \times n} .
$$

Step 1. Formula (1) formula (2) are used to calculate the coverage rate of one pixel over each sensor node.

Step 2. Formula (4) is used to calculate the joint coverage rate of the pixel over each sensor node.

Step 3. Repeat procedures of Steps 1 and 2 to calculate the joint coverage rate of each pixel over each sensor node.

Step 4. Formula (5) is used to calculate the regional coverage rate of the region.

(c) The Number of Neighboring Nodes of the Network. Take the mean value of the number of neighboring nodes of all sensor nodes, indicating the connectivity of the network. The concept of the neighboring node is that if $T_{i j}=1$, then the sensor node $S_{i}$ and the sensor node $S_{j}$ are the neighboring nodes of each other. We set that there are $N$ neighboring nodes for the node $S_{i}$; the number of the neighboring nodes of the network is

$$
\text { Neigh }=\frac{\sum_{k=1}^{N} \text { Neighbor }_{k}}{2 N} .
$$

(d) The Communication Strength of Network. Take the mean value of the communication strength of all wireless sensor nodes, indicating the network communication performance. Depending on the communication model, the communication strength of the node $S_{i}$ is (which is the communication strength of the wireless sensor network)

$$
\text { Trans }=\frac{\sum_{i=1}^{N} T\left(S_{i}, S_{j}\right)}{2 N} .
$$

(e) The Number of Islands. The entire network can be divided into unconnected regions, indicating the connectivity of the network. The concept of the joint node is that if $T_{i j}>0$, then the sensor node $S_{i}$ and the sensor node $S_{j}$ are the joint nodes of each other. The $N+M$-dimensional adjacency matrix $G=$ $\left\{g_{i j}\right\}$ is written, in which

$$
g_{i j}= \begin{cases}1 & T_{i j}>0 \\ 0 & T_{i j}=1 .\end{cases}
$$

According to the rules above, $G=\left(a_{1}, a_{2}, \ldots, a_{N+M}\right)$ is converted to $G^{\prime}=\left(a_{1}, a_{2}, \ldots, a_{N+M}\right)^{T}$, where $G^{\prime}$ is referred to as the connection matrix, the characteristic of which is, in the row vector with module value $>1$, the working nodes corresponding to the row of nonzero factor are connected; the working nodes corresponding to the different row vector with the module value $>1$ are not connected. Therefore, the $\operatorname{rank}\left(G^{\prime}\right)$ connecting the matrix $G^{\prime}$ indicates the number of the connection areas in the separate regions formed by the nodes, which is the number of islands.

(f) The Minimum Cost Model. $Z_{a}$ is the sensor node, $C_{\text {cost }}$ is the cost of every sensor node, and $m$ is the number of the sensor nodes:

$$
\min U=\sum_{a \in m}\left(Z_{a} * C_{\text {cost }}\right)
$$

4.1. Constrained Optimization Problem. The solution to multiple target planning is the main research area of multiple target planning problem. For the characteristics of the multiple target planning itself, seeking the solution to the multiple target planning is also seeking the noninferior solution. The main solution methods are evaluation function method, constraint method, interaction, voting analytic hierarchy process, power function method, fuzzy method, maximum entropy, and human intelligence method. Moreover, there are also some improved hereditary algorithms, particle swarm optimization and other human intelligence algorithms.

The key of the wireless sensor network deployment optimization is, through optimizing the position of the sensor nodes, the coverage and connectivity performance of the network comprised of nodes can reach the optimal state and meet the requirement of both the coverage of the road network and the connectivity of the network. And, thus, the wireless sensor network deployment optimization oriented towards the traffic information collection is a constrained optimization problem:

$$
\begin{aligned}
\max F= & \max \left[\frac{1-\prod_{s_{i} \in s}\left(1-P_{\mathrm{cov}}\left(x, y, s_{i}\right)\right)}{m \times n}\right] \\
& \times \max \left[\frac{\sum_{i=1}^{N} T\left(S_{i}, S_{j}\right)}{N}\right] .
\end{aligned}
$$

But formula (10) does not reflect the cost of the sensor network building; formula (9) should be introduced to our final model. Meanwhile, through comparison, the geometric weighting method can make a better result.

The first step is to uniform the dimension and standardize the target functions $f_{i}(i=1,2, \ldots, m)$. Solve the maximum value $f_{i, \max }$ and the minimum value $f_{i \text {,min }}$ of the target functions, standardizing the target function by means of the function $\varphi_{i}(x)=\left(f_{i}(x)-f_{i, \text { min }}\right) /\left(f_{i, \text { max }}-f_{i, \text { min }}\right)$.

The second step is to give a group of weight coefficients corresponding to the target function $\varphi_{i}$ based on different significance of the target function; in the formula, $w_{i}>0(i=$ $1,2, \ldots, m)$ and $\sum_{i=1}^{m} w_{i}=1$. The linear weighting method is to give a group of nonnegative number $w_{i}(i=1,2, \ldots, m)$ 
corresponding to the target $f_{i}$ for different significance of the target function; then the linear combination is made of $f_{i}$ and $w_{i}$ to obtain the following evaluation function:

$$
u(f(x))=\sum_{i=1}^{m} w_{i} f_{i}(x) .
$$

The multiple target solution problem is converted to be

$$
(M 1) \begin{cases}\min & \sum_{i=1}^{m} w_{i} f_{i}(x) \\ \text { s.t. } & x \in X .\end{cases}
$$

$X$ is the constraint set of the multiobject function, which ensures that the solution of the objective function is a noninferior solution of the multiobject programming.

Specifically, when $w_{i}=1 / m(i=1,2, \ldots, m)$, the evaluation function is

$$
\frac{1}{m} f_{1}(x)+\frac{1}{m} f_{2}(x)+\cdots+\frac{1}{m} f_{m}(x) \text {. }
$$

This is the arithmetic mean made of $f_{1}(x), f_{2}(x), \ldots$, $f_{m}(x)$. Similar to the arithmetic mean, the geometric mean is made of $f_{1}(x), f_{2}(x), \ldots, f_{m}(x)$, obtaining

$$
f_{1}(x)^{1 / m} \times f_{2}(x)^{1 / m} \times \cdots \times f_{m}(x)^{1 / m} .
$$

Generally, when $w_{i}(i=1,2, \ldots, m)$ are not all equivalent, the evaluation function is taken:

$$
u(f(x))=\prod_{i=1}^{m} f_{i}(x)^{w_{i}}
$$
be

Then the multiple target solution problem is converted to

$$
(M 2) \begin{cases}\min & \prod_{i=1}^{m} f_{i}(x)^{w_{i}} \\ \text { s.t. } & x \in X .\end{cases}
$$

The unconstrained optimization problem equivalent to the constrained optimization problem above is

$$
(M 2) \begin{cases}\min \quad & {\left[\max \left(\frac{1-\prod_{s_{i} \in s}\left(1-P_{\text {cov }}\left(x, y, s_{i}\right)\right)}{m \times n}\right)^{w_{1}} \times \max \left(\frac{\sum_{i=1}^{N} T\left(S_{i}, S_{j}\right)}{N}\right)^{w_{2}} \times \min \left(\sum_{a \in m} Z_{a} * C_{\text {cost }}\right)^{w_{3}}\right]} \\ \text { s.t. } \quad & R_{\text {area }}(S) \geq 0.95 \\ & \operatorname{Neigh}_{i}>0 \\ & g_{i}(X)>0, \quad i=1,2, \ldots, n .\end{cases}
$$

In the formula, $g_{i}(X)>0$ is the constrained condition determined by the requirement of the road network coverage and the network connection.

(a) Coverage Requirement. The sensor networks coverage level reaches to some certain value. In the formula, $R_{\text {area }}(s)$ is the requirement of the road networks for the coverage level, which should be more than $95 \%$.

\section{(b) Connectivity Requirement.}

The number of the neighboring nodes of any sensor node is larger than 0 .

The number of the neighboring nodes of any sink node is larger than 0 .

In the island formed by the networks, the number of islands not including the sink node is 0 .

The problem can be classified as the optimization problem. As the problem model is complex, thus we can employ a new intelligent optimization algorithm, the chemical reaction optimization algorithm, to solve it.

\section{Chemical Reaction Optimization Algorithm}

The chemical reaction optimization algorithm simulates the chemical molecules' change and migration which prompt the molecules to move towards the optimal node in the solution space. A chemical reaction occurs in a vessel containing a certain number of molecules. Every molecule has one molecule solution structure, dynamic energy, and potential energy. The chemical reaction optimization uses the potential energy to simulate the value of optimization function: $\mathrm{PE}_{\omega}=f(\omega)$, where $f$ is the optimization function and $\omega$ is the molecule solution structure. These reaction modes include the following: nondestructive on-wall collision, decomposition reaction, nondestructive molecule collision, and combination reaction. These basic reaction modes all have different algorithms of changing the molecule solution structure.

5.1. Algorithm Design. In the nondestructive on-wall collision, one molecule strikes the vessel wall. We can make small perturbation by randomly changing the position of any two of the binary strings, changing the existing solution $\omega$ to $\omega^{\prime}$; for example, consider the following. 
(a) On-Wall Ineffective Collision. This operator only changes the solution structure of one molecule when the nondestructive on-wall collision occurs. Therefore, in the algorithm, the existing molecule $\omega$ should be the input, outputting a new molecule $\omega^{\prime}$. The main design purpose of the operator is to make a small-scale exploration on the potential energy surface, and thus it should change the molecule solution structure in a small scale:

$$
\underbrace{0010100110000101}_{\omega} \longrightarrow \underbrace{0000100110001101}_{\omega^{\prime}}
$$

(b) Decomposition. A molecule $\omega$ is decomposed into two new molecules $\omega_{1}^{\prime}$ and $\omega_{2}^{\prime}$; the new molecules have a big difference from the original molecule. In order to achieve this, we adopt the following method. Consider the $i$-bit of $\omega$ as $\omega(i)$. If it equals 1 , then its value is copied to the same position of $\omega_{1}^{\prime}$ and the rest bits of $\omega_{1}^{\prime}$ are set at random. Meanwhile, the value of $\omega(i)$ is copied to the same position of $\omega_{2}^{\prime}$ and the rest bits of $\omega_{2}^{\prime}$ are set at random. With this method, $\omega_{1}^{\prime}$ and $\omega_{2}^{\prime}$ will have clear difference. This mechanism will make CRO algorithm jump out of the local minimum value; for example, consider the following.

This operator applies the existing two molecules $\omega$ to produce two new molecules $\omega_{1}^{\prime}$ and $\omega_{2}^{\prime}$. This operator can help the molecule jump out of the regional minimum value. In the chemical reaction optimization, we divide $\omega$ into two parts, $\omega_{1}^{\prime}$ and $\omega_{2}^{\prime}$, and then complement randomly the missing part of the molecules to produce two new molecule solution structures:

$$
\begin{aligned}
& \underbrace{0010 \underline{100110000 \underline{1} 01}}_{\omega} \\
& \longrightarrow \underbrace{10 \underline{10} \underline{101} \underbrace{10}_{110110 \underline{10}} \text { AND } \underbrace{0000100100000100}_{\omega_{2}^{\prime}} .}_{\omega_{1}^{\prime}} .
\end{aligned}
$$

(c) Intermolecular Ineffective Collision. In the intermolecular ineffective collision, the two molecules $\omega_{1}$ and $\omega_{2}$ strike each other. We add perturbation to the two molecules simultaneously to obtain the new molecules $\omega_{1}^{\prime}$ and $\omega_{2}^{\prime}$. In order to achieve this, we employ the same method for $\omega_{1}^{\prime}$ and $\omega_{2}^{\prime}$ as that in the nondestructive on-wall collision. For example, consider the following.

This operator randomly changes the two existing $\omega_{1}$ and $\omega_{2}$ to produce new molecules $\omega_{1}^{\prime}$ and $\omega_{2}^{\prime}$ when the nondestructive molecules collide with each other. This operator randomly combines and exchanges the existing molecules, so different parts of the potential optimum can possibly be changed into the same molecule solution structure. In the chemical reaction optimization, the operator involves two steps. Step one: the comparison is made of the two molecule solution structures for selecting one commonly owned node. Step two: the four parts with the node as the boundary will be crossed and exchanged to produce a new molecule solution structure:

$$
\begin{aligned}
& \underbrace{[00 \underline{10100110000101]}}_{\omega_{1}} \operatorname{AND} \underbrace{[0010100110000101]}_{\omega_{2}} \\
& \longrightarrow \underbrace{[00 \underline{000100110001101]}}_{\omega_{1}^{\prime}} \text { AND } \underbrace{[0010100010010101]}_{\omega_{2}^{\prime}} \text {. }
\end{aligned}
$$

(d) Synthesis. We produce the existing two molecules $\omega_{1}$ and $\omega_{2}$ into a new molecule $\omega^{\prime}$. In order to differentiate $\omega^{\prime}$ from $\omega_{1}$ and $\omega_{2}$, we make a XOR of $\omega_{1}$ and $\omega_{2}$ to produce the new molecule $\omega^{\prime}$. For example, this operator combines two existing molecules $\omega_{1}$ and $\omega_{2}$ to produce a new molecule $\omega^{\prime}$. In the chemical reaction optimization, the operator is rather similar to the nondestructive collision operator. Differently, after choosing the commonly owned node in step one, the four parts will pair with each other two by two to calculate the occupation rate of the total resources. The new molecule solution structure is the structure occupying the minimum rate of the total resources:

$$
\begin{aligned}
& \underbrace{[0010100110000101]}_{\omega_{1}} \oplus \underbrace{[0100000110101001]}_{\omega_{2}} \\
& \longrightarrow \underbrace{[0110100000101100]}_{\omega^{\prime}} .
\end{aligned}
$$

Figure 1 illustrates the flowchart of CRO algorithm; the main procedures of the algorithm are described as follows.

Step 1. In the initialization, we initialize a set of molecules with the size of popsize. The objective function is the PE of the molecule. The initial KE of every molecule is set to the value of initial $\mathrm{KE}$.

Step 2. In each iteration, we decide the collision type according to whether it is a unimolecular or an intermolecular reaction. When it is a unimolecular reaction, we adopt the on-wall ineffective collision or decomposition. When it is an intermolecular reaction, we adopt the intermolecular ineffective collisions or synthesis.

Step 3. Calculate the value of the objective function and check if it is the new minimum point.

Step 4. The iteration process continues until a stopping criterion is satisfied. We output the best-so-far solution in the final stage.

Stopping Criterion. The program will be stopped when the iteration number reaches the predetermined maximum iteration number.

\section{Simulation Results}

In MATLAB simulation environment, simulate the network structural layout of the base and the density optimization process, verify the performance of CRO algorithm in solving the wireless sensor networks deployment, and compare it with 


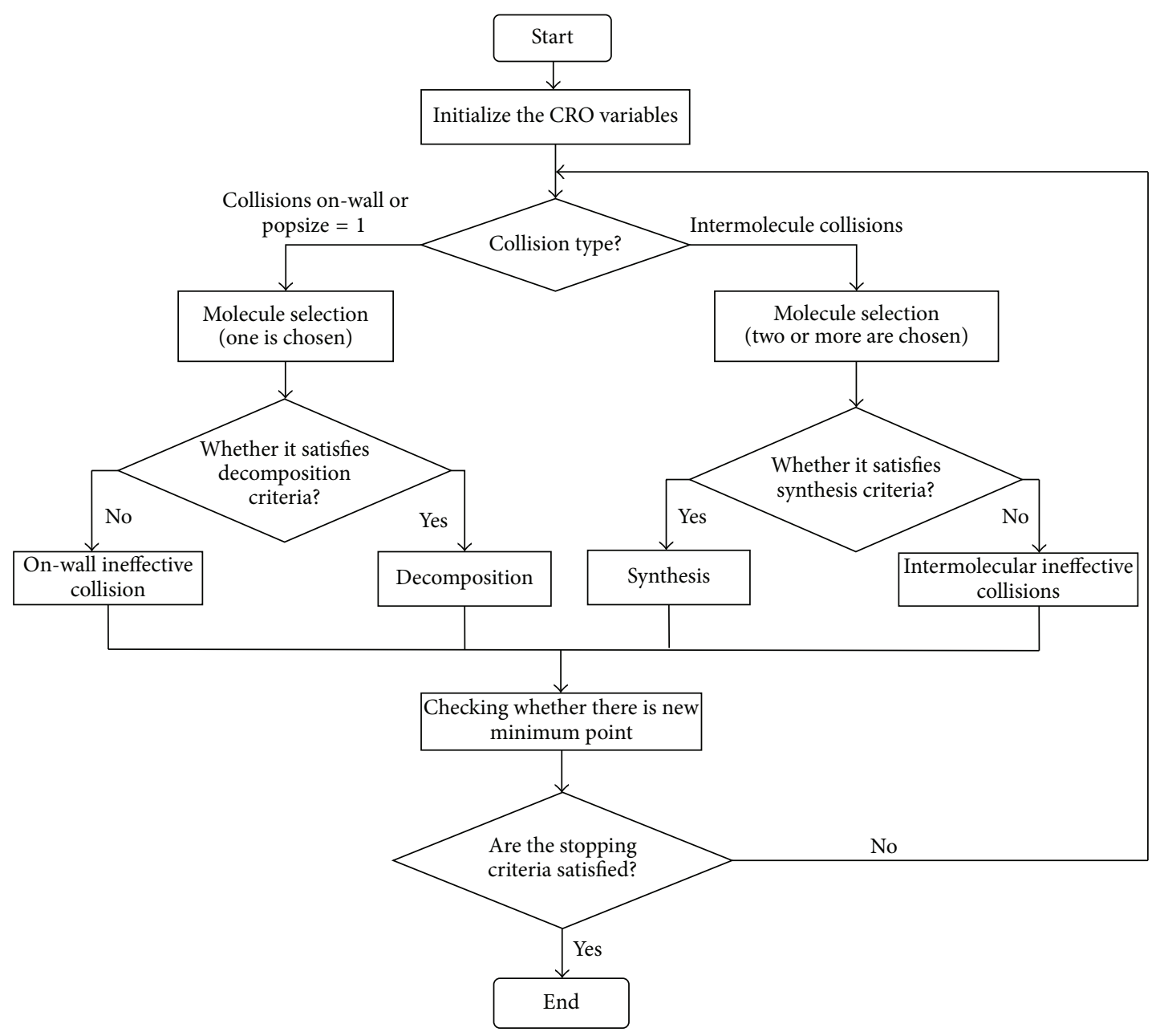

FIgURE 1: The flowchart of CRO algorithm.

PSO (particle swarm optimization) algorithm and BGPSO (bacterial foraging particle swarm optimization) algorithm.

The simulating experiment conditions are that the nodes are randomly deployed in a range of $500 \mathrm{~m} \times 500 \mathrm{~m}$. The main detection criteria for locating problem are Average Localization Error. The experiment results are shown in Figures 3 5. After multiple and repeated experiments, the controlling parameters are set as follows: the node perception radius is $\mathrm{CO}=20 \mathrm{~m}$, the communication distance of the node $R_{1}=20 \mathrm{~m}$, the weight of networks area coverage $w_{1}=0.6$, the weight of the networks communication strength $w_{2}=0.25$, the weight of the networks connectivity $w_{3}=0.15$, and the number of sensor nodes $n_{1}=100$.

Figure 2 is the neighboring relation schema between nodes, in which the red circle is the sensor node and the blue connecting line is the communication link between nodes. If the communication can be made between two nodes, then the blue connecting line can be established between them as the neighboring nodes of each other. From Figure 2, we can see that the nodes are connecting and communicating with each other, and thus no separate nodes and information island are produced.

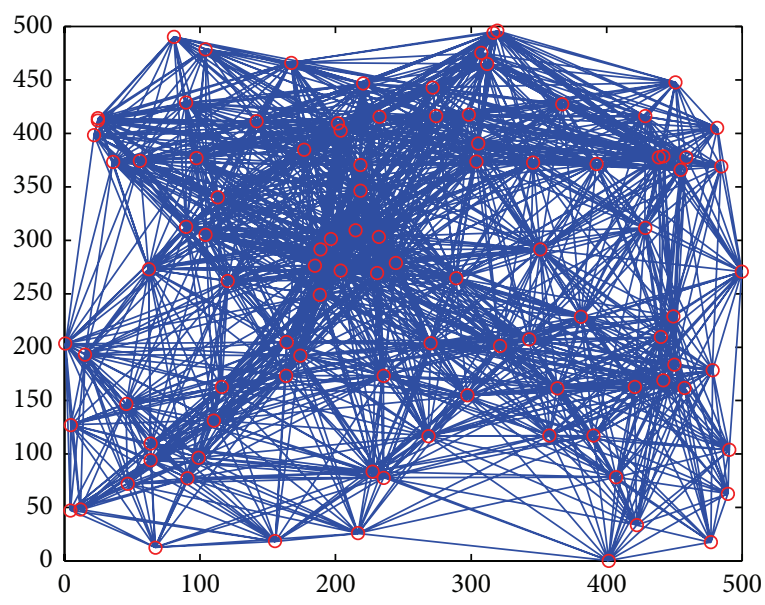

FIgURE 2: The neighboring relation schema between nodes.

Figure 3 is the area coverage schema of nodes, in which the red asterisk is the sensor node, the blue circle is the sensor radius of the node, and the area located in the blue circle is 

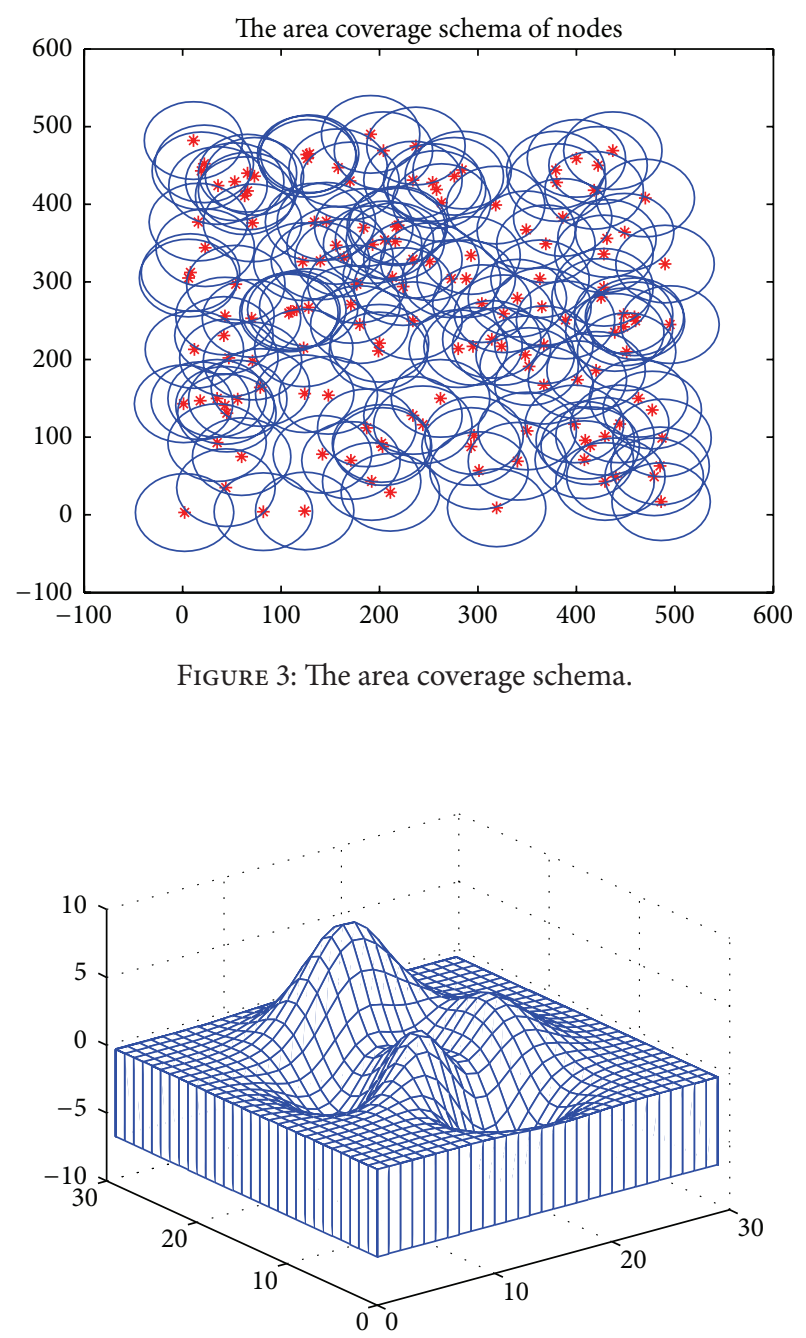

FIgure 4: The CRO algorithm.

the area sensed and detected by the node. We hope to make a better deployment of the node by means of CRO algorithm to cover most parts of the area.

Figure 4 is the chemical reaction optimization algorithm diagram. In the algorithm, we employ different operators to make the potential energy of the overall model function approach the low area as much as possible (the global optimum), and thus, compared with other algorithms, it can always find the global optimum. As the algorithm contains the nondestructive on-wall collision which can speed up the movement and change of the molecule and the operating speed of the whole procedure and the decomposition and combination operators which can intensify the movement between molecules, thus it can jump out of the local optimal trap. Overall, the CRO algorithm is a powerful new intelligence algorithm with high speed.

Figure 5 is the relation schema between the number of iterations and the perception radius. In the simulation, we compare CRO algorithm with other two commonly used intelligent algorithms PSO and BGPSO. The perception radius of the node is from $8 \mathrm{~m}$ to $9.8 \mathrm{~m}$ with the step length
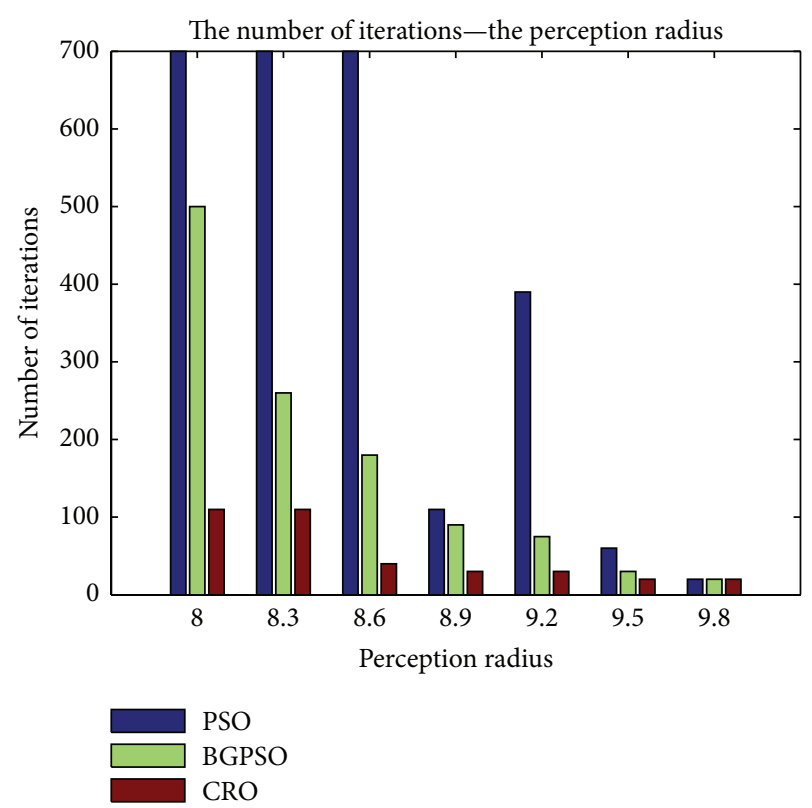

FIGURE 5: Relation schema between the number of iterations and the perception radius.

as $0.3 \mathrm{~m}$. Record the number of iterations as the constrained conditions in formula (17) are met. Moreover, in order to avoid the procedure to be elected for long-time ineffective running, when the number of iterations of the procedure reaches 700 times, this operation would be stopped, and the number of iterations is set to be 700 . From Figure 5, we can find that $\mathrm{CRO}$ algorithm can meet the constrained conditions of the system under different perception radius in the case of a small number of iterations; that is, the area coverage is larger than $95 \%$ and the nodes can communicate with each other without generation of islands. Therefore, we can conclude that the efficiency of CRO algorithm is much higher than that of BGPSO and PSO algorithm.

Table 1 is the checklist of fitness function optimum, where five simulation experiments are conducted of CRO algorithm, BGPSO algorithm, and PSO algorithm, respectively. In every experiment, the optimal fitness function value of the three algorithms should be recorded to indicate the best networks deployment and density optimization effect obtained. From Table 1, we can see in the five experiments that the optimal mean value of the fitness function of CRO algorithm is 11.656, the optimal mean value of the fitness function of BGPSO algorithm is 10.924, the optimal mean value of the fitness function of PSO algorithm is 10.38, and the optimal mean value of the fitness function of CRO algorithm is higher than that of BGPSO algorithm and PSO algorithm by 0.732 and 1.276 , respectively.

The main reason is that CRO algorithm is a comprehensive algorithm, which contains multiple operators such as decomposition, combination, and nondestructive on-wall collision, capable of changing the coordinate position of the node to a great extent when the procedure is in operation, which can help to find the global optimum in a better and more rapid way. 
TABLE 1: Comparison schema of fitness function optimum.

\begin{tabular}{lccc}
\hline Experiment number & PSO & BGPSO & CRO \\
\hline Experiment 1 & 10.45 & 10.92 & 11.61 \\
Experiment 2 & 10.37 & 10.95 & 11.67 \\
Experiment 3 & 10.52 & 10.89 & 11.59 \\
Experiment 4 & 10.29 & 10.83 & 11.69 \\
Experiment 5 & 10.27 & 11.03 & 11.72 \\
\hline
\end{tabular}

\section{Conclusion}

For the reasonable deployment of the wireless sensor, we establish the wireless sensor network deployment optimization model, applying CRO algorithm to solve the deployment optimization. The experiment results show that CRO algorithm outperforms PSO algorithm in solving the wireless sensor networks deployment optimization of traffic information collection, which can effectively optimize the wireless sensor network layout of traffic information collection to improve the comprehensive evaluation value of networks performance by $12.29 \%$; CRO algorithm can improve the disadvantage of PSO algorithm in sinking into the optimal local solution easily to obtain a better wireless sensor network layout.

\section{Conflict of Interests}

The authors declare that there is no conflict of interests regarding the publication of this paper.

\section{Acknowledgments}

This work is supported by a project funded by the National Natural Science Foundation of China (Grant no. 61304187), funded by China Postdoctoral Science Foundation (Grant no. 2014M560040), funded by the Scientific Research Fund of SiChuan Provincial Science and Technology Department of China (Grant no. 2015JY0213), funded by Scientific Research Fund of SiChuan Provincial Education Department of China (Grant no. 15TD0038), and funded by the Foundation of Chengdu University (no. 2014XJZ21).

\section{References}

[1] K. C. Sinha, "Can technologies cure transportation," in Proceedings of the 6th International Conference on Application of Advance Technology in Transportation Engineering, pp. 242-246, August 2000.

[2] J. Alder, "Dynamic route guidance and in vehicle systems," Intelligent Transport Systems, vol. 2, no. 3, pp. 125-132, 2000.

[3] J. Jia, J. Chen, G. Chang, and Z. Tan, "Energy efficient coverage control in wireless sensor networks based on multi-objective genetic algorithm," Computers \& Mathematics with Applications, vol. 57, no. 11-12, pp. 1756-1766, 2009.

[4] J. Chen, J. Li, S. He, Y. Sun, and H.-H. Chen, "Energy-efficient coverage based on probabilistic sensing model in wireless sensor networks," IEEE Communications Letters, vol. 14, no. 9, pp. 833-835, 2010.
[5] G. Lei and L. Shixiang, "Coal resources demand evaluation based on support vector regression with improved quantumbehaved PSO," Journal of Convergence Information Technology, vol. 7, no. 1, pp. 405-412, 2012.

[6] D. E. Goldberg, Genetic Algorithms in Search, Optimization and Machine Learning, Addison-Wesley, Reading, Mass, USA, 1989.

[7] E. Aarts and J. Korst, Simulated Annealing and Boltzmann Machines: A Stochastic Approach to Combinatorial Optimization and Neural Computing, John Wiley \& Sons, 1989.

[8] K. Wu, Y. Gao, F. Li, and Y. Xiao, "Lightweight deploymentaware scheduling for wireless sensor networks," Mobile Networks and Applications, vol. 10, no. 6, pp. 837-852, 2005.

[9] T. Niknam, "An approach based on particle swarm optimization for optimal operation of distribution network considering distributed generators," in Proceedings of the 32nd Annual Conference on IEEE Industrial Electronics (IECON '06), pp. 633637, IEEE, Paris, France, November 2006.

[10] A. Y. S. Lam and V. O. K. Li, "Chemical-reaction-inspired metaheuristic for optimization," IEEE Transactions on Evolutionary Computation, vol. 14, no. 3, pp. 381-399, 2010.

[11] A. Y. S. Lam, J. Xu, and V. O. K. Li, "Chemical Reaction Optimization for population transition in peer-to-peer live streaming," in Proceedings of the IEEE Congress on Evolutionary Computation (CEC '10), pp. 1-8, Barcelona, Spain, July 2010.

[12] J. Xu, A. Y. S. Lam, and V. O. K. Li, "Chemical reaction optimization for task scheduling in grid computing," IEEE Transactions on Parallel and Distributed Systems, vol. 22, no. 10, pp. 1624-1631, 2011.

[13] J. Xu, A. Y. S. Lam, and V. O. K. Li, "Chemical reaction optimization for the grid scheduling problem," in Proceedings of the IEEE International Conference on Communications (ICC '10), pp. 470-475, May 2010.

[14] G. Jun, "Research on wireless sensor network coverage algorithm and simulation," Computational Simulation, vol. 27, no. 9, pp. 146-149, 2010. 


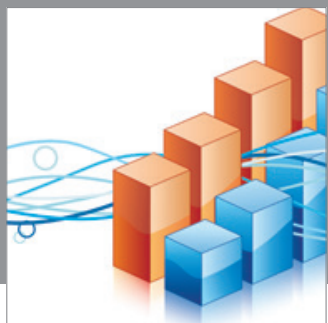

Advances in

Operations Research

mansans

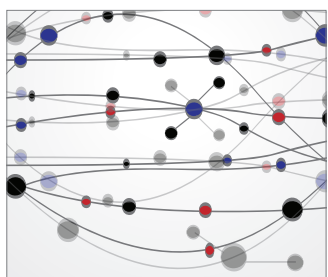

The Scientific World Journal
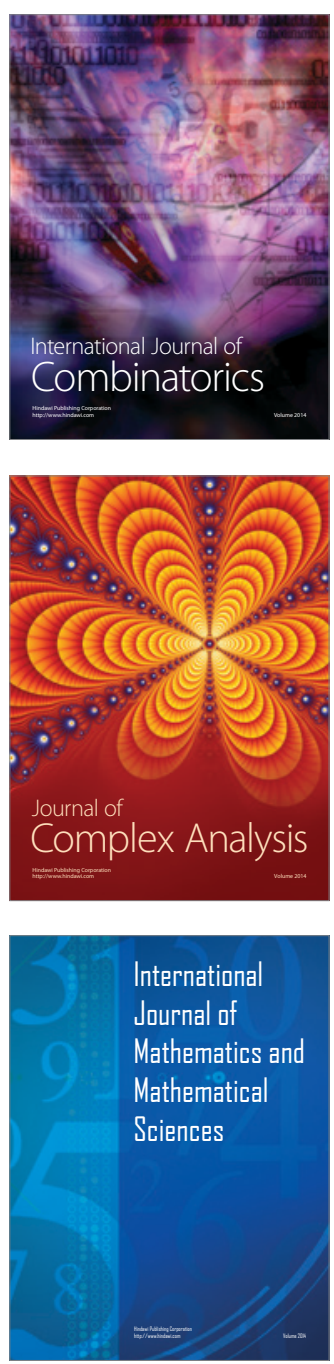
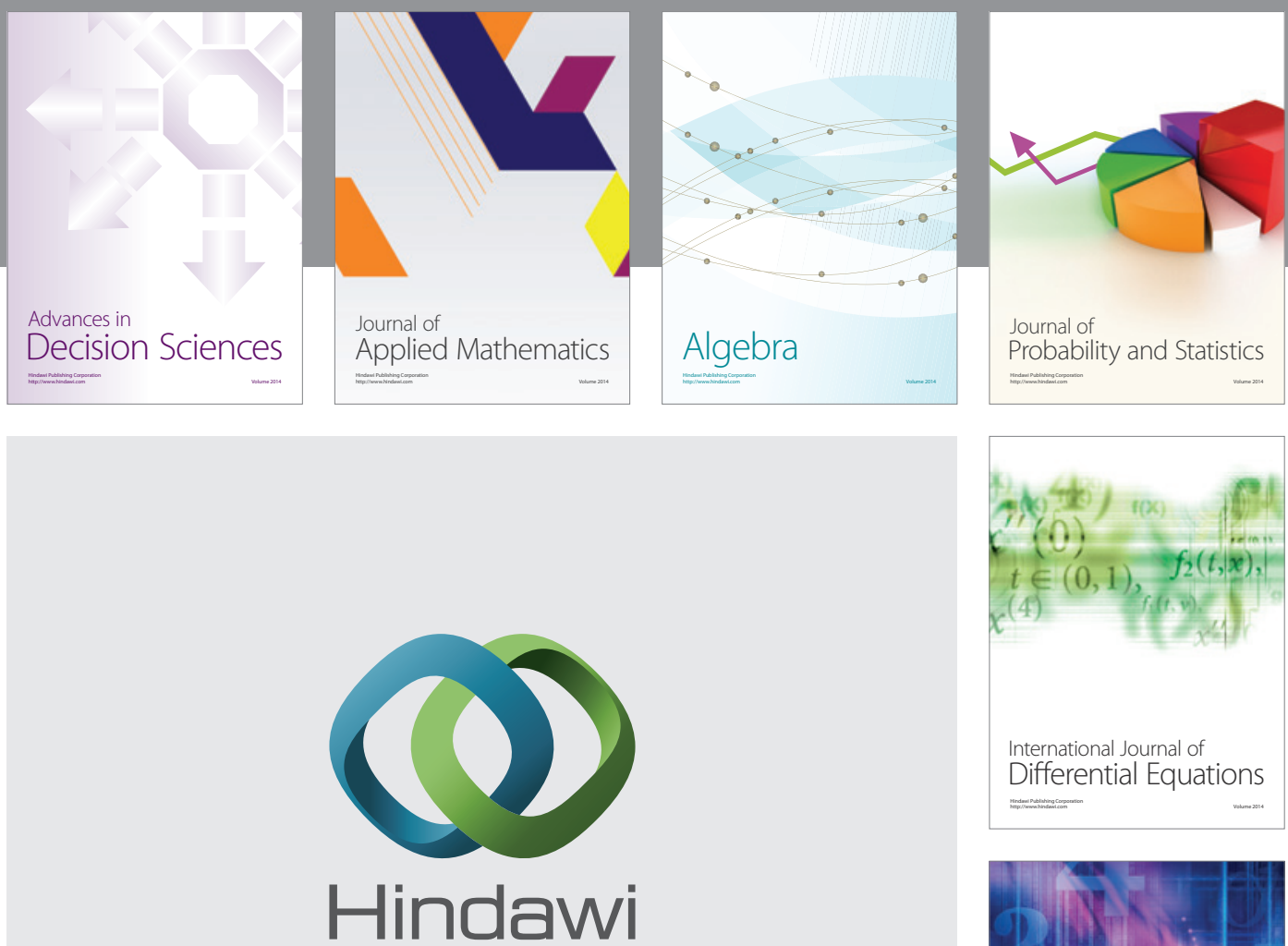

Submit your manuscripts at http://www.hindawi.com
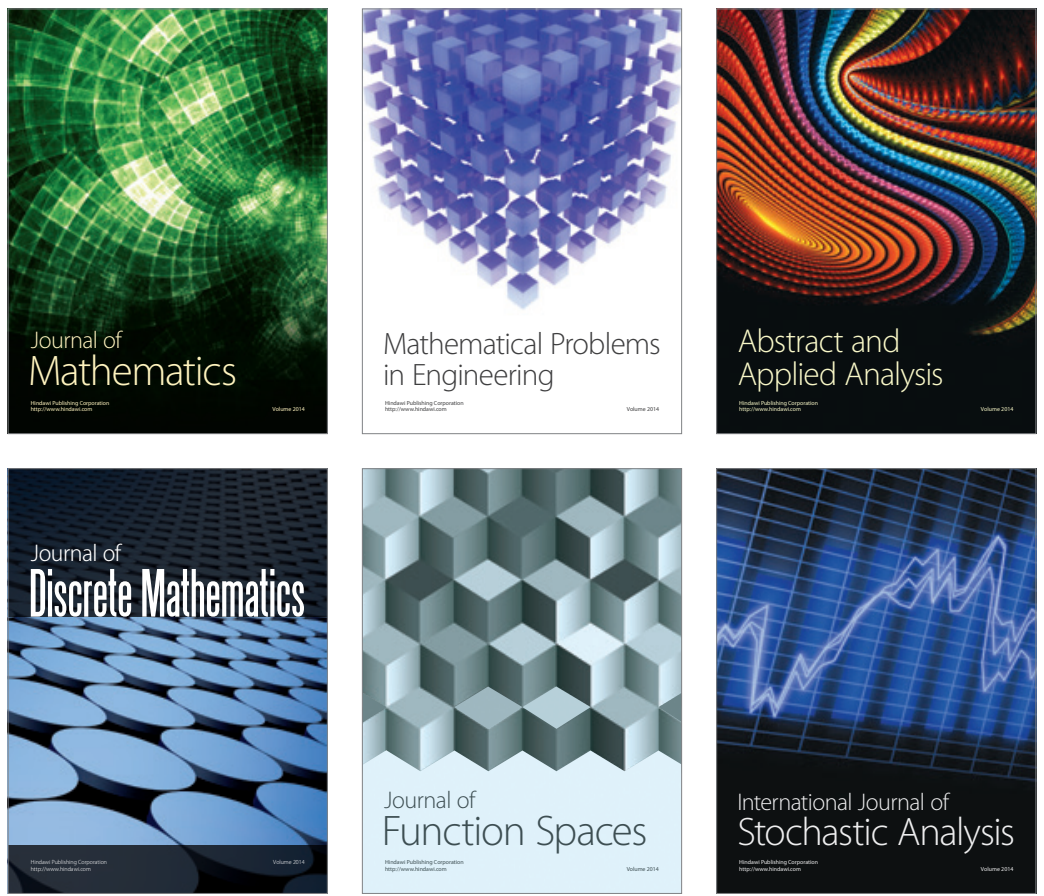

Journal of

Function Spaces

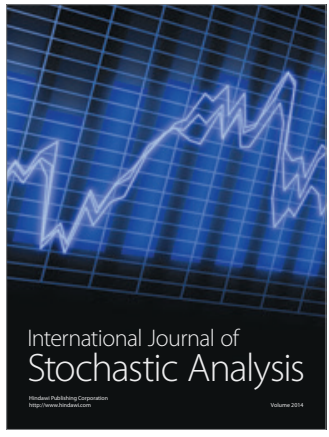

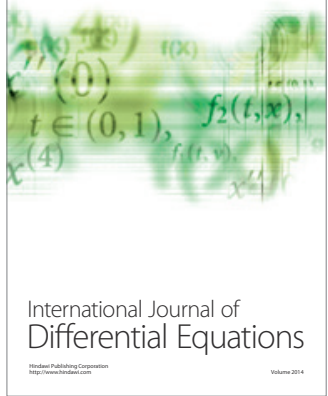
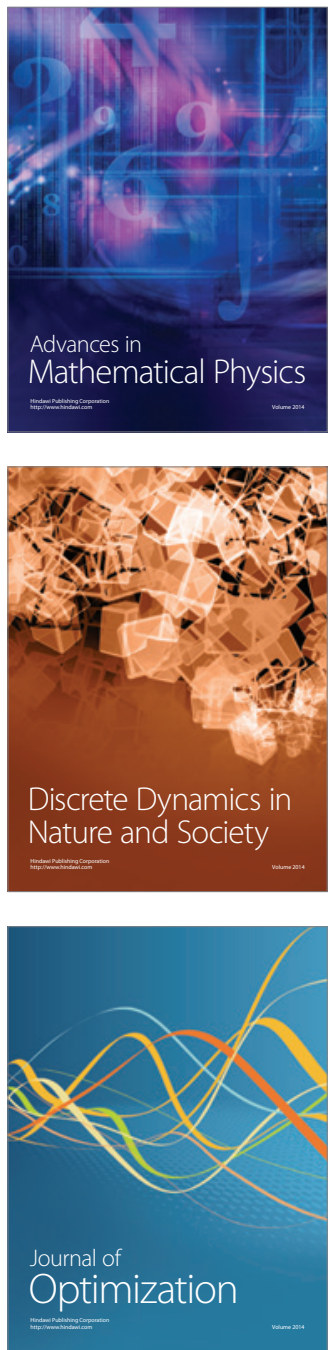